\title{
The local disturbance decoupling problem with stability for nonlinear systems
}

\author{
Leo van der WEGEN and Henk NIJMEIJER \\ University of Twente, Department of Applied Mathematics, P.O. Box 217, 7500 AE Enschede, The Netherlands
}

Received 8 August 1988

Revised 29 October 1988

\begin{abstract}
In this paper the Disturbance Decoupling Problem with Stability (DDPS) for nonlinear systems is considered. The DDPS is the problem of finding a feedback such that after applying this feedback the disturbances do not influence the output anymore and $x=0$ is an exponentially stable equilibrium point of the feedback system. For systems that can be decoupled by static state feedback it is possible to define (under fairly mild assumptions) a distribution $\Delta_{\mathrm{s}}^{*}$ which is the nonlinear analogue of the linear $\mathscr{V}_{\mathrm{s}}^{*}$, the largest stabilizable controlled invariant subspace in the kernel of the output mapping, and to prove that the DDPS is locally solvable if and only if the disturbance vector fields are contained in $\Delta_{s}^{*}$.
\end{abstract}

Keywords: Nonlinear control system; disturbance decoupling with stability; controlled invariant distribution.

\section{Introduction}

Since the appearance of Wonham's well-known book on the geometric approach to linear control problems [13] much attention has been paid to the further development of this theory for linear systems. Also, a considerable amount of research has been done on the generalization of this theory to nonlinear systems using a differential-geometric approach. This has led to local solutions for several well-known design problems like the Disturbance Decoupling Problem and the Noninteracting Control Problem. A good overview of results in this area up to 1985 can be found in Isidori [5]. In recent years a couple of articles on stabilizability of nonlinear systems have appeared, e.g. Charlet [3], Aeyels [1], Tsinias [11], Jurdjevic \& Quinn [7].

However, nor the book [5], neither these articles pay attention to design problems in connection with stability of the feedback system, in contrast to Isidori \& Grizzle [6], Byrnes \& Isidori [2] and Van der Wegen \& Nijmeijer [12].

In [6], Isidori \& Grizzle derive some negative results concerning the solution of the Noninteracting Control Problem with Stability. They show that this problem is not solvable by applying static feedback if the fixed internal dynamics inherent to the noninteracting requirement is unstable. In [2], Byrnes \& Isidori solve the Disturbance Decoupling Problem (DDP) with BIBO-stability for systems which are (exponentially) minimum phase.

As a matter of fact, the minimum-phase requirement is rather strong. For linear systems it comes down to requiring that $\mathscr{V}^{*}$, the maximal controlled invariant subspace in the kernel of the output mapping, and $\mathscr{V}_{\mathrm{s}}{ }^{*}$, the largest stabilizable controlled invariant subspace in $\mathscr{V}^{*}$, coincide. Under this assumption, the conditions for solvability of the DDP and the Disturbance Decoupling Problem with Stability (DDPS) for linear systems are the same, whereas in general conditions for solvability of the latter problem are stronger.

The generalization of $\mathscr{V}^{*}$ for nonlinear systems is $\Delta^{*}$, the largest locally controlled invariant distribution in the kernel of the output mapping. Necessary and sufficient conditions for the (local) solvability of the DDP are stated in terms of $\Delta^{*}$ (see [5]). These conditions are similar to the ones in the linear case. In this paper we will give a nonlinear analogue of $\mathscr{V}_{\mathrm{s}}^{*}$, called $\Delta_{\mathrm{s}}^{*}$, and solve the DDPS for systems that can be decoupled by static state feedback. It will appear that the conditions for solvability of the DDPS look very much alike the linear and nonlinear case. 
In Section 2 we will give the problem formulation, the definition of $\Delta_{\mathrm{s}}^{*}$ and the solution to the DDPS. Some examples are worked out in Section 3, and in Section 4 our results are compared to those of Byrnes and Isidori. We end up with an appendix where some standard results on hyperbolic vector fields are summarized.

\section{The local disturbance decoupling problem with stability}

Consider the analytic SISO control system

$$
\dot{x}=f(x)+g(x) u+e(x) d, \quad y=h(x), x \in \mathbb{R}^{n}, d \in \mathbb{R},
$$

with $f(0)=0$ and $h(0)=0$.

We want to solve the Disturbance Decoupling Problem with Stability (DDPS) for (2.1), i.e. find a controlled invariant distribution $\Delta$ and a feedback

$$
u=\alpha(x)+\beta(x) v, \quad \alpha(0)=0, \beta(x) \text { invertible }
$$

such that

$$
e \in \Delta \subset \operatorname{ker} \mathrm{d} h
$$

and $x=0$ is an exponentially stable equilibrium point of the system

$$
\dot{x}=(f+g \alpha)(x) \text {. }
$$

In general, it is hard to find a global solution. Therefore, we will be satisfied if we can find $\Delta$ and a feedback defined locally around $x=0$ such that $\Delta$ is locally controlled invariant and $x=0$ is locally exponentially stable.

We make the following assumptions:

(A1) The DDP is locally solvable for (2.1).

(This is equivalent with the condition that $e \in \Delta^{*}$.)

(A2) The system (2.1) is accessible in a neighborhood of $x=0$, i.e.

$$
\operatorname{sp}\langle f, g \mid \operatorname{sp}\{f, g\}\rangle(x)=T_{x} \mathbb{R}^{n}
$$

for all $x$ in a neighborhood of $x=0$ (see Sussmann \& Jurdjevic [10]).

(A3) The function $A(x)$ defined by

$$
A(x)=L_{g} L_{f}^{\rho(x)} h(x)
$$

is nonzero in a neighborhood of $x=0$. Here the characteristic number $\rho(x)$, the smallest integer such that

$$
L_{g} L_{f}^{k} h(x)=0, \quad k<\rho(x),
$$

and

$$
L_{g} L_{f}^{\rho(x)} h(x) \neq 0
$$

is assumed to be constant in a neighborhood of $x=0$. Note that (A3) implies that $\rho:=\rho(x)$ is finite. 
To stress the resemblance with the linear case as well as the differences we will in short sketch the solution of the DDPS in the linear case.

Consider the SISO linear system

$$
\dot{x}=A x+B u+E d, \quad y=C x, \quad x \in \mathbb{R}^{n}, d \in \mathbb{R}^{r} .
$$

Suppose that (A1), (A2), and (A3) hold for this system. Note that (A1) is equal to the condition that Im $E \subset \mathscr{V}^{*}$, while (A2) is equivalent to saying that (2.9) is controllable. Under these assumptions we can find a feedback

$$
u=F x+G v, \quad G \text { invertible, }
$$

such that $(A+B F) \mathscr{V}^{*} \subset \mathscr{V}^{*}$. It is well known that the eigenvalues of $\left.(A+B F)\right|_{\mathscr{r}^{*} \text {, the so-called }}$ transmission zeros of the system, are independent of the choice of $F$. Hence, there exists a unique subspace of $\mathscr{V}^{*}$ spanned by the (generalized) eigenvectors corresponding to the exponentially stable eigenvalues of the matrix $A+B F$. This subspace is denoted by $\mathscr{V}_{\mathrm{s}}^{*}$. Note that $\mathscr{V}_{\mathrm{s}}^{*}$ is controlled invariant, because $\mathscr{V}_{\mathrm{s}}^{*}$ is $(A+B F)$-invariant. Assume now that

$$
\operatorname{Im} E \subset \mathscr{V}_{\mathrm{s}}^{*}
$$

then we can solve the DDPS for (2.9) by choosing a feedback (2.10) such that $(A+B F) \mathscr{V}_{\mathrm{s}}^{*} \subset \mathscr{V}_{\mathrm{s}}^{*}$ and $A+B F$ is an asymptotically stable matrix, because by (A2), the induced system on $\mathbb{R}^{n} / \mathscr{V}_{\mathrm{s}}^{*}$ is controllable.

We look at the solution of this problem in a different way now. The linear subspaces $\mathscr{V}^{*}$ and $\mathscr{V}_{\mathrm{s}}^{*}$ can be considered as integral manifolds through $x=0$ of the flat distributions $\Delta_{\mathscr{F}^{*}} \cong \mathscr{V}^{*}$ and $\Delta_{\mathscr{V}^{*}} \cong \mathscr{V}_{\mathrm{s}}^{*}$, respectively. The manifold $\mathscr{V}_{s}^{*}$ is invariant under the vector field $(A+B F) x$, for $x=0$ is an equilibrium point of this vector field. Since $\Delta_{\mathscr{V}^{*}}$ is spanned by constant vector fields, this distribution is necessarily invariant under any vector field of the form $(A+B F)^{i} B G, i=0, \ldots, n-1$. This implies that $X^{t}\left(\mathscr{V}_{\mathrm{s}}^{*}\right)$

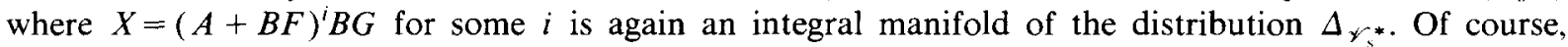
$X^{\mathrm{t}}\left(\mathscr{V}_{\mathrm{s}}^{*}\right)=x+\mathscr{V}_{\mathrm{s}}^{*}$ for some $x \in \mathbb{R}^{n}$ (depending on $t$ ). As a matter of fact, it is possible to construct the foliation $\left\{x+\mathscr{V}_{\mathrm{s}}^{*} \mid x \in \mathbb{R}^{n}\right\}$ from the integral manifold $\mathscr{V}_{\mathrm{s}}^{*}$ through $x=0$. By controllability,

$$
\operatorname{sp}\left\{(A+B F)^{i} B G \mid i=0, \ldots, n-1\right\}=T_{x} \mathbb{R}^{n}
$$

for any $x \in \mathbb{R}^{n}$, and so it is possible to find independent vector fields $X_{1}, \ldots, X_{n-k}$ in $\left\{(A+B F)^{i} B G \mid i=\right.$ $0, \ldots, n-1\}$ that are transversal to the manifold $\mathscr{V}_{\mathrm{s}}^{*}$ of dimension $k$. (See the appendix for the definitions of independence and transversality.) The set

$$
\left\{X_{n-k}^{t_{n-k}} \cdots \cdots \circ X_{1}^{t_{1}}\left(\mathscr{V}_{s}^{*}\right) \mid t_{1}, \ldots, t_{n-k} \in \mathbb{R}\right\}
$$

defines a foliation on $\mathbb{R}^{n}$. Note that the order of the $X_{i}$ in (2.12) does not matter, since $\left[X_{i}, X_{j}\right]=0$ for $j$, $i=1, \ldots, n-k$. This foliation (2.12) is the same as the foliation $\left\{x+\mathscr{V}_{s}^{*} \mid x \in \mathbb{R}^{n}\right\}$, for the $X_{i}$ are constant vector fields. This implies that it is possible to find the distribution $\Delta_{r_{*}^{*}}$ from the foliation (2.12) that was defined using only the integral manifold $\mathscr{F}_{s}^{*}$ through $x=0$ and a particular set of vector fields transversal to this integral manifold.

It is in this way that we are going to construct the generalization of $\mathscr{V}_{\mathrm{s}}^{*}$ for nonlinear systems.

We return to the nonlinear system (2.1) now. Note that (A3) implies that $S^{*}\left(\Delta^{*}\right)$, the largest local controllability distribution in $\Delta^{*}$ (and so, in $\mathrm{ker} \mathrm{d} h$ ) is equal to the zero distribution (see Nijmeijer [8]). Again by assumption (A3) it is possible to choose new coordinates in the following way. Let

$$
\xi_{1}=\left[h(x), L_{f} h(x), \ldots, L_{f}^{o} h(x)\right]^{\mathrm{T}} .
$$

If $\rho+1<n$, then choose an extra set of coordinates $\xi_{2}$. It is well known (see Isidori [5], Chapter 4) that by choosing

$$
\beta(x)=(A(x))^{-1}, \quad \alpha(x)=-(A(x))^{-1} L_{f}^{\rho+1} h, \quad u=\alpha(x)+\beta(x) \tilde{v},
$$


the system (2.1), (2.2) with $d \equiv 0$ becomes in the new coordinates

$$
\begin{aligned}
& \dot{\xi}_{1}=A_{1} \xi_{1}+B_{1} \tilde{v} . \\
& \dot{\xi}_{2}=\bar{f}_{2}\left(\xi_{1}, \xi_{2}\right)+\bar{g}_{2}\left(\xi_{1}, \xi_{2}\right) \tilde{v}, \\
& y=\xi_{11} .
\end{aligned}
$$

with

$$
A_{1}=\left(\begin{array}{cccc}
0 & 1 & & 0 \\
\vdots & & \ddots & \\
\vdots & & & 1 \\
0 & \ldots & \ldots & 0
\end{array}\right), \quad B_{1}=\left(\begin{array}{c}
0 \\
\vdots \\
\vdots \\
0 \\
1
\end{array}\right)
$$

Obviously, $\left(A_{1}, B_{1}\right)$ is a controllable pair. Hence it is possible to apply another feedback

$$
\tilde{v}=N \xi_{1}+v
$$

such that $M:=\mathrm{A}_{1}+B_{1} N$ is an unstable matrix, i.e. $\sigma(M) \subset \mathbb{C}^{+}:=\{s \in \mathbb{C} \mid \operatorname{Re} s>0\}$.

Now the system (2.15), (2.17) has the form

$$
\begin{aligned}
& \dot{\xi}_{1}=M \xi_{1}+B_{1} v, \\
& \dot{\xi}_{2}=\bar{f}_{2}\left(\xi_{1}, \xi_{2}\right)+\bar{g}_{2}\left(\xi_{1}, \xi_{2}\right) N \xi_{1}+\bar{g}_{2}\left(\xi_{1}, \xi_{2}\right) v, \\
& y=\xi_{11} .
\end{aligned}
$$

Next, choose new coordinates $\left(x_{1}^{\mathrm{T}}, x_{2}^{\mathrm{T}}\right)^{\mathrm{T}}$ as follows:

$$
x_{1}=\xi_{2}, \quad x_{2}=\xi_{1} .
$$

Then the system (2.18) including the disturbances has the following form:

$$
\begin{aligned}
& \dot{x}_{1}=\hat{f}\left(x_{1}, x_{2}\right)+\hat{g}_{1}\left(x_{1}, x_{2}\right) N x_{2}+\hat{g}_{1}\left(x_{1}, x_{2}\right) v+e_{1}\left(x_{1}, x_{2}\right) d, \\
& \dot{x}_{2}=M x_{2}+\hat{g}_{2} v, \\
& y=\hat{h}\left(x_{2}\right) .
\end{aligned}
$$

Note that it follows from (2.18) that $\hat{\mathrm{g}}_{2}$ is constant. Moreover, by (A1), e belongs to $\Delta^{*}=\operatorname{sp}\left\{\partial / \partial x_{1}\right\}=$ $\operatorname{sp}\left\{\partial / \partial \xi_{2}\right\}$. It follows directly from (2.20) that

$$
\left[\tilde{f}, \Delta^{*}\right] \subset \Delta^{*}, \quad\left[\tilde{g}, \Delta^{*}\right] \subset \Delta^{*}
$$

where

$$
\tilde{f}(x)=\left(\begin{array}{c}
\hat{f}\left(x_{1}, x_{2}\right)+\hat{g}_{1}\left(x_{1}, x_{2}\right) N x_{2} \\
M x_{2}
\end{array}\right), \quad \tilde{g}(x)=\left(\begin{array}{c}
\hat{g}_{1}\left(x_{1}, x_{2}\right) \\
\hat{g}_{2}
\end{array}\right) .
$$

Equation (2.20) will be our starting point for the definition of a possible generalization of $\mathscr{V}_{*}^{*}$. At this point, we make another assumption:

(A4) The zero dynamics of the system has an hyperbolic equilibrium at $x=0$.

This implies in particular that the set of eigenvalues of the matrix

$$
F=\frac{\partial \tilde{f}}{\partial x}(0)=\left(\begin{array}{cc}
\frac{\partial \hat{f}}{\partial x_{1}} & \frac{\partial \hat{f}}{\partial x_{2}}+\hat{g}_{1}\left(x_{1}, x_{2}\right) N \\
0 & M
\end{array}\right)_{x_{1}=0, x_{2}=0}
$$


has no intersection with the imaginary axis. Note that this is a restriction on $f(x)$ and $g(x)$ only, since $N$ can be chosen arbitrarily. In that case there exist uniquely determined analytic stable and unstable manifolds through $x=0$ for the system $\dot{x}=\tilde{f}(x)$ (see the appendix). Since $\Delta^{*} \cap \operatorname{sp}\{g\}=0$ we have that the feedback is uniquely determined on the integral manifold $M_{0}$ of $\Delta^{*}$ through $x=0$ (see [6]). This implies that the vector field $\hat{f}$ is uniquely determined on $M_{0}$. Therefore, the stable manifold $S_{0}$ through $x=0$ is completely contained in $M_{0}$, because $S_{0}$ as well as $M_{0}$ is invariant under $\tilde{f}$. As noted earlier we will, if possible, construct a distribution $\Delta_{s}^{*}$ now (which will a priori depend on the choice of the vector $N$ ) that plays the role of $\mathscr{V}_{\mathrm{s}}^{*}$ in this nonlinear setting.

Definition 2.1. A stable distribution $\Delta_{\mathrm{s}}$ is a distribution that is locally controlled invariant, contained in $\Delta^{*}$, and for which the linearization of the dynamics restricted to the leaf of $\Delta_{\mathrm{s}}$ through $x=0$ is asymptotically stable.

Obviously, the class of stable distributions is nonempty, since the zero distribution is contained in this class. First, we try to construct a stable distribution $\Delta_{\mathrm{s}}$ for which $S_{0}$ is the leaf through $x=0$. Suppose that the vectorfield $X$ belongs to $D$, where $D$ denotes the set of vector fields

$$
D=\left\{\operatorname{ad}_{\tau_{l}} \operatorname{ad}_{\tau_{l-1}} \cdots \operatorname{ad}_{\tau_{1}} \tau_{0} \mid l \in \mathbb{N}, \tau_{0}, \ldots, \tau_{l} \in\{\tilde{f}, \tilde{g}\}\right\} .
$$

Then the manifold $X^{t}\left(S_{0}\right)$ should be an integral manifold of this distribution $\Delta_{\mathrm{s}}$ (for, if $\Delta_{\mathrm{s}}$ is invariant under $\tilde{f}$ and $\tilde{g}$, then it is invariant under all Lie brackets of these vector fields). Assume that $S_{0}$ has dimension $k$. By the accessibility condition (A2) it is possible to find, locally around $x=0$, independent vector fields $X_{1}, \ldots, X_{n-k}$ in $D$ that are transversal to $S_{0}$. As a matter of fact, none of these $X_{i}$ is equal to $f$, since $f(0)=0$. Once the order of the $X_{i}$ 's is fixed, the set

$$
\left\{X_{n-k}^{t_{n-k}} \circ X_{n-k-1}^{t_{n-k-1}} \circ \cdots \circ X_{1}^{t_{1}}\left(S_{0}\right) \mid-\varepsilon \leq t_{i} \leq \varepsilon, 1 \leq i \leq n-k\right\}
$$

defines a foliation in a neighborhood of $x=0$. To explain this, we construct the foliation (2.25) for a one-dimensional $S_{0}$ in $\mathbb{R}^{3}$; the general case follows along the same lines. In this special case there exist locally around $x=0$ two independent vector fields $X_{1}$ and $X_{2}$ in the set $D$ (see (2.24)) that are transversal to $\mathrm{S}_{0}$. Obviously, the set $\left\{X_{1}^{t_{1}}\left(\mathrm{~S}_{0}\right) \mid-\varepsilon \leq t_{1} \leq \varepsilon\right\}$ defines (locally around $x=0$ ) a foliation on a two-dimensional manifold $L$ in $\mathbb{R}^{3}$. Consider a point $p$ outside $L$, but sufficiently close to $x=0$. Then there exists a $t_{2}$ such that $q:=X_{2}^{-t_{2}}(p)$ lies on one of the leaves of $\left\{X_{1}^{t_{1}}\left(S_{0}\right) \mid-\varepsilon \leq t_{1} \leq \varepsilon\right\}$, say $S_{1}$. Hence $p \in X_{2}^{t_{2}}\left(S_{1}\right)=$ $X_{2}^{t_{2}} \circ X_{1}^{t_{1}}\left(S_{0}\right)$. Since $p$ is arbitrary, the foliation (2.25) is defined on a neighborhood of $x=0$ in $\mathbb{R}^{3}$. Note that it depends on the order of the $X_{i}$ 's. The foliation (2.25) will be called stable, because it is generated by the stable manifold $S_{0}$ (that is associated with the stable eigenvalues of the matrix $F$ in (2.23)). This terminology is in accordance with that given in Palis \& de Melo [9]. (Note that a stable distribution is not just the distribution associated with a stable foliation (cf. Definition 2.1).) If the foliation (2.25) is invariant under $\tilde{f}$ and $\tilde{g}$, it defines a distribution $\Delta_{s}$ that is invariant under these vector fields. In that case the foliation (2.25) does not depend on the order of the $X_{i}$ 's.

Lemma 2.1. Suppose that the foliation (2.25) is invariant under $\tilde{f}$ and $\tilde{g}$. Then it uniquely defines a stable distribution $\Delta_{s}$. Moreover, $\Delta_{s}$ is independent of the choice of $N$ in (2.17).

Proof. The uniqueness of $\Delta_{s}$ (for a fixed $N$ ) follows immediately from the definition of the stable manifold $S_{0}$ and the construction of the foliation (2.25). The inclusion $\Delta_{\mathrm{s}} \subset \Delta^{*}$ follows from the fact that both $\Delta_{\mathrm{s}}$ and $\Delta^{*}$ are invariant under $\tilde{f}$ and $\tilde{g}$ and $S_{0} \subset M_{0}$. Consider eq. (2.20) now with $d \equiv 0$ and assume that $x_{1}=\left(x_{11}^{\mathrm{T}}, x_{12}^{\mathrm{T}}\right)^{\mathrm{T}}$ is chosen such that

$$
\Delta_{\mathrm{s}}=\operatorname{sp}\left\{\frac{\partial}{\partial x_{11}}\right\} \text {. }
$$


Then we have, by the invariance of $\Delta_{s}$ under $\tilde{f}$ and $\tilde{g}$,

$$
\begin{aligned}
& \dot{x}_{11}=\hat{f_{11}}\left(x_{11}, x_{12}, x_{2}\right)+\hat{g}_{11}\left(x_{11}, x_{12}, x_{2}\right) N x_{2}+\hat{g}_{11}\left(x_{11}, x_{12}, x_{2}\right) v, \\
& \dot{x}_{12}=\hat{f_{12}}\left(x_{12}, x_{2}\right)+\hat{g}_{12}\left(x_{12}, x_{2}\right) N x_{2}+\hat{g}_{12}\left(x_{12}, x_{2}\right) v, \\
& \dot{x}_{2}=M x_{2}+\hat{g}_{2} v, \\
& y=\hat{h}\left(x_{2}\right) .
\end{aligned}
$$

It follows immediately from (2.27) that $\Delta_{\mathrm{s}}$ is invariant under $\tilde{f}$ and $\tilde{g}$ for every feedback that leaves $\Delta^{*}=\operatorname{sp}\left\{\partial / \partial x_{11}, \partial / \partial x_{12}\right\}$ invariant, whether this feedback is linear or nonlinear. This is implied by the fact that every such feedback

$$
v=\phi\left(x_{2}\right)+w, \quad \phi\left(x_{2}\right)=0,
$$

with $\phi$ arbitrary only depends on $x_{2}$. By choosing $v=-N x_{2}+\tilde{N} x_{2}+w$ with $\tilde{N}$ an arbitrary vector for which $A_{1}+B_{1} \tilde{N}$ is unstable, it is easy to see that $\Delta_{\mathrm{s}}$ does not depend on the choice of $N$ in (2.17).

In the linear case, $\mathscr{V}_{s}^{*}$ is the maximal controlled invariant subspace in ker $C$ such that the dynamics of the system restricted to this subspace is asymptotically stable. Motivated by this linear paradigm, we investigate if there exists a maximal element in the class of stable distributions, i.e. we search for a locally controlled invariant distribution $\Delta_{5}^{*}$ contained in $\Delta^{*}$ for which the linearization of the dynamics restricted to the leaf of $\Delta_{s}^{*}$ through $x=0$ is asymptotically stable and that contains all stable distributions.

In case the foliation (2.25) is invariant under $\tilde{f}$ and $\tilde{g}$, the stable distribution $\Delta_{s}$ that is defined by this foliation is maximal, since $S_{0}$ has maximal dimension (for $S_{0}$ is the stable manifold through $x=0$ of the vector field $\tilde{f}$ in (2.22)). Hence, the maximal stable distribution $\Delta_{\mathrm{s}}^{*}$ equals $\Delta_{\mathrm{s}}$.

The next question is of course if $\Delta_{s}^{*}$ exists when $(2.25)$ is not invariant under $\tilde{f}$ and $\tilde{g}$. Clearly the construction of a foliation like the one given in (2.25) may be repeated for any stable manifold $S$ contained in $S_{0}$ (see the appendix). If the foliation generated by $S$ is invariant under $\tilde{f}$ and $\tilde{g}$, then it can be proved along the lines of Lemma 2.1 that there exists a stable distribution $\Delta(S)$ associated with it. Since the zero distribution is stable, we can define $\bar{\Delta}$, as the involutive sum of all stable distributions. Clearly, the integral manifold $\tilde{S}$ of $\bar{\Delta}_{\mathrm{s}}$ through $\mathrm{x}=0$ is stable, maximal and contains all integral manifolds $S$ through $x=0$ of the stable distributions $\Delta(S)$ that are contained in $\bar{\Delta}_{s}$. Along the lines of Lemma 2.1 we can prove now that $\bar{\Delta}_{\mathrm{s}}$ is a uniquely defined stable distribution independent of the choice of $N$ in (2.17). In fact $\bar{\Delta}_{\mathrm{s}}$ equals $\Delta_{\mathrm{s}}^{*}$, since the integral manifold $\tilde{S}$ of $\bar{\Delta}_{\mathrm{s}}$ through $x=0$ is the largest manifold that generates a stable distribution.

We have proved the following lemma.

Lemma 2.2. There exists a uniquely defined maximal stable distribution $\Delta_{\leftarrow}^{*}$. Moreover, if $\Delta^{*}$ is invariant under $\tilde{f}$ and $\tilde{g}$, then so is $\Delta^{*}$.

Corollary. For systems with $m$ inputs and $m$ outputs $\Delta_{5}^{*}$ can be defined along the same lines as above if we replace assumption (A3) by the following:

(A3') The characteristic numbers $\rho_{1}(x), \ldots, \rho_{m}(x)$ are constant and the decoupling matrix $A(x)$ is invertible in a neighborhood of $x=0$.

Remarks. (i) Unfortunately, the existence result on $\Delta_{\mathrm{s}}^{*}$ does not give a method to construct this distribution in practice.

(ii) Although the definition of $\Delta_{s}^{*}$ is fairly analogous to the construction of $\mathscr{V}_{s}^{*}$ there is an important difference, mainly due to the fact that we have the extra (strong!) requirement that $\Delta_{s}^{*}$ should be invariant under $\tilde{g}$. This is the reason why the dimension of $\Delta_{\mathrm{s}}^{*}$ can be strictly less than the number of stable eigenvalues of the matrix $F$ in $(2.23)$. 
By now, the solution to the DDPS for (2.1) is straightforward. For convenience, we choose new coordinates $x=\left(z_{1}^{\mathrm{T}}, z_{2}^{\mathrm{T}}\right)^{\mathrm{T}}$ such that

$$
\Delta_{s}^{*}=\operatorname{sp}\left\{\frac{\partial}{\partial z_{1}}\right\}
$$

This yields, instead of (2.19),

$$
\begin{aligned}
& \dot{z}_{1}=\tilde{\tilde{f}}_{1}\left(z_{1}, z_{2}\right)+\tilde{\tilde{g}}_{1}\left(z_{1}, z_{2}\right) v+\tilde{\tilde{e}}_{1}\left(z_{1}, z_{2}\right) d, \\
& \dot{z}_{2}=\tilde{\tilde{f}}_{2}\left(z_{2}\right)+\tilde{\tilde{g}}_{2}\left(z_{2}\right) v+\tilde{\tilde{e}}_{2}\left(z_{1}, z_{2}\right) d, \\
& y=\tilde{\tilde{h}}\left(z_{2}\right) .
\end{aligned}
$$

Assume:

(A5) $\left(\left(\partial \tilde{\tilde{f}} / \partial z_{2}\right)(0), \tilde{\tilde{g}}_{2}(0)\right)$ is a controllable pair.

(A6) $e \in \Delta_{\mathrm{s}}^{*}$.

Assumption (A6) implies that $\tilde{\tilde{e}}_{2}$ is identically equal to zero and (A5) that there exists a linear feedback

$$
v=G z_{2}+w
$$

such that the system (2.30), (2.31),

$$
\begin{aligned}
& \dot{z}_{1}=\tilde{\tilde{f}}\left(z_{1}, z_{2}\right)+\tilde{\tilde{g}}_{1}\left(z_{1}, z_{2}\right) G z_{2}+\tilde{\tilde{g}}_{1}\left(z_{1}, z_{2}\right) w+\tilde{\tilde{e}}_{1}\left(z_{1}, z_{2}\right) d, \\
& \dot{z}_{2}=\tilde{\tilde{f}}\left(z_{2}\right)+\tilde{\tilde{g}}_{2}\left(z_{2}\right) G z_{2}+\tilde{\tilde{g}}_{2}\left(z_{2}\right) w, \\
& y=\tilde{\tilde{h}}\left(z_{2}\right),
\end{aligned}
$$

is locally exponentially stable around $z=0$ (see [7]). Notice that $\Delta_{\mathrm{s}}^{*}$ is invariant under both the drift vector field

$$
\check{f}(z)=\left(\begin{array}{c}
\tilde{\tilde{f}}\left(z_{1}, z_{2}\right)+\tilde{\tilde{g}}_{1}\left(z_{1}, z_{2}\right) G z_{2} \\
\tilde{\tilde{f}}\left(z_{2}\right)+\tilde{\tilde{g}}_{2}\left(z_{2}\right) G z_{2}
\end{array}\right) \quad \text { and } \quad \check{g}(z)=\left(\begin{array}{c}
\tilde{\tilde{g}}_{1}\left(z_{1}, z_{2}\right) \\
\tilde{\tilde{g}}_{2}\left(z_{2}\right)
\end{array}\right) \text {. }
$$

We conclude that $z=0$ is an exponentially stable equilibrium point of the system $\dot{z}=\check{f}(z)$ and that $e \in \Delta_{\mathrm{s}}^{*},\left[\breve{f}, \Delta_{\mathrm{s}}^{*}\right] \subset \Delta_{\mathrm{s}}^{*},\left[\check{g}, \Delta_{\mathrm{s}}^{*}\right] \subset \Delta_{\mathrm{s}}^{*}$, and $\Delta_{\mathrm{s}}^{*} \subset \operatorname{ker} \mathrm{d} h$; hence the DDPS for (2.1) is solved.

For convenience, we summarize the result in the following theorem.

Theorem 2.1. Consider the system (2.1). Assume that (A1) up to (A5) hold. Then the DDPS for (2.1) is locally solvable if and only if $e \in \Delta_{s}^{*}$.

Proof. The only assertion that has to be proved is the 'only if' part. Assume that the DDPS is solvable. It follows from [5, p. 131] that there exists a distribution $\Delta$ that is invariant under $\tilde{f}$ and $\tilde{g}$ such that

$e \in \Delta \subset \operatorname{ker} \mathrm{d} h$.

Since the feedback system has $x=0$ as a (locally) exponentially stable equilibrium point, the integral manifold of $\Delta$ through $x=0$ is certainly stable. Hence, by definition of $\Delta_{\mathrm{s}}^{*}, \Delta \subset \Delta_{\mathrm{s}}^{*}$, and consequently $e \in \Delta_{\mathrm{s}}^{*}$.

Obviously, Theorem 2.1 is also valid for systems with as many inputs as outputs for which assumption $\left(\mathrm{A} 3^{\prime}\right)$ holds and for systems with an arbitrary number of disturbances. 
Remark. Consider the following controllable linear system with nonlinear disturbances:

$$
\begin{aligned}
& \dot{x}=A x+B u+e(x) d, \\
& y=C x .
\end{aligned}
$$

Suppose that (A1), (A2) and (A3) hold. Choose $u=F x+v$ in such a way that $(A+B F) \mathscr{V}^{*} \subset \mathscr{V}^{*}$ and the matrix $A+B F$ has no eigenvalues on the imaginary axis (i.e. (A4) is valid). Then $\Delta_{\mathrm{s}}^{*}$ is just the flat distribution $\Delta_{\mathscr{V}^{*}} \cong \mathscr{V}_{s}^{*}$. This implies that the DDPS for this system is solvable if and only if $e \in \Delta_{s}^{*}$ (i.e. (A6)). (Note that assumption (A5) is induced by the controllability of the system.) This result is in agreement with Theorem 2.2 in [12].

\section{Examples}

In this section we give two examples to illustrate the theory given in the previous section. In the first one the dimension of $\Delta_{\mathrm{s}}^{*}$ is equal to the number of stable eigenvalues of the matrix $F$ in $(2.23)$, whereas in the second one this dimension is strictly less.

Example 3.1. Consider the analytic control system

$$
\dot{x}=f(x)+g(x) u+e(x) d, \quad y=h(x),
$$

with

$$
f(x)=\left(\begin{array}{c}
-x_{1}+x_{3} \\
x_{2}+x_{3} \\
\left(x_{2}+1\right) x_{3}
\end{array}\right), \quad g(x)=\left(\begin{array}{c}
x_{1}\left(1+x_{1}\right) \\
0 \\
1+x_{1}
\end{array}\right), \quad e(x)=\left(\begin{array}{c}
x_{2}^{2} \mathrm{e}^{x_{3}} \\
0 \\
0
\end{array}\right), \quad h(x)=x_{3} .
$$

Now $L_{g} h=x_{1}+1 \neq 0$ in a neighborhood of $x=0$. Hence $\rho=0$ and $\Delta^{*}=\operatorname{sp}\left\{\partial / \partial x_{1}, \partial / \partial x_{2}\right\}$. Choose

$$
u=\frac{1}{1+x_{1}}\left[-\left(x_{2}+1\right) x_{3}+x_{3}+v\right] \text {. }
$$

Notice that (3.3) is well defined in a neighborhood of $x=0$. Now equations (3.1)-(3.3) yield

$$
\dot{x}=\tilde{f}(x)+\tilde{g}(x) v+e(x) d, \quad y=h(x),
$$

with

$$
\tilde{f}(x)=\left(\begin{array}{c}
-x_{1}-x_{1} x_{2} x_{3}+x_{3} \\
x_{2}+x_{3} \\
x_{3}
\end{array}\right), \quad \tilde{g}(x)=\left(\begin{array}{c}
x_{1} \\
0 \\
1
\end{array}\right) .
$$

Obviously, $\Delta^{*}$ is invariant under $\tilde{f}$ and $\tilde{g}$. In this case

$$
F=\frac{\partial \tilde{f}}{\partial x}(0)=\left(\begin{array}{ccc}
-1 & 0 & 1 \\
0 & 1 & 1 \\
0 & 0 & 1
\end{array}\right)
$$

so the stable eigenspace of $F$ is $\operatorname{sp}\left\{e_{1}\right\}$. Moreover, since

$$
\left[\tilde{f,} \frac{\partial}{\partial x_{1}}\right]=\left(\begin{array}{c}
1+x_{2} x_{3} \\
0 \\
0
\end{array}\right) \in \operatorname{sp}\left\{\frac{\partial}{\partial x_{1}}\right\}, \quad\left[\tilde{g}, \frac{\partial}{\partial x_{1}}\right]=-\frac{\partial}{\partial x_{1}} .
$$

it is obvious that $\Delta_{s}^{*}$ equals $\operatorname{sp}\left\{\partial / \partial x_{1}\right\}$ in this case. And so, for this simple example it is not necessary to calculate the foliation (2.25) starting from the stable manifold $\left\{x \mid x_{2}=x_{3}=0\right\}$. Note that $e \in \Delta_{s}^{*}$ thus 
(A6) is satisfied. Easy calculations show that assumptions (A2) and (A5) are fulfilled. Choose another feedback

$$
v=-4 x_{2}-4 x_{3}+w
$$

then the system (3.4), (3.5), (3.8) has the form

$$
\dot{x}=\tilde{\tilde{f}}(x)+\tilde{g}(x) w+e(x) d, \quad y=h(x),
$$

with

$$
\tilde{\tilde{f}}(x)=\left(\begin{array}{c}
-x_{1}+x_{3}-x_{1} x_{2} x_{3}-4 x_{1} x_{2}-4 x_{1} x_{3} \\
x_{2}+x_{3} \\
-4 x_{2}-3 x_{3}
\end{array}\right)
$$

It is easy to verify that $\Delta_{s}^{*}$ is invariant under $\tilde{\tilde{f}}$ and that $x=0$ is an exponentially stable equilibrium point of the equation $\dot{x}=\tilde{f}(x)$. This implies that the local DDPS for (3.1), (3.2) is solvable by applying the feedback (3.3), (3.8).

Example 3.2. Consider the system (3.1) with

$$
f(x)=\left(\begin{array}{c}
-2 x_{1} \\
-x_{2}+x_{4} \\
x_{3}-x_{2} x_{3} \\
3 x_{4}
\end{array}\right), \quad g(x)=\left(\begin{array}{r}
1 \\
-1 \\
1 \\
1
\end{array}\right), \quad h(x)=x_{4}, \quad e(x) \text { arbitrary }
$$

In this case $L_{g} h=1$, hence $\rho=0$ and $\Delta^{*}=\operatorname{sp}\left\{\partial / \partial x_{1}, \partial / \partial x_{2}, \partial / \partial x_{3}\right\}$. Oviously, $\Delta^{*}$ is invariant under $f$ and $g$ and

$$
\frac{\partial f}{\partial x}(0)=\left[\begin{array}{rrrr}
-2 & 0 & 0 & 0 \\
0 & -1 & 0 & 1 \\
0 & 0 & 1 & 0 \\
0 & 0 & 0 & 3
\end{array}\right]
$$

and the stable manifold through $x=0$ is $S_{0}=\left\{x \mid x_{3}=x_{4}=0\right\}$. Define

$$
\begin{aligned}
& k:=[f, g]=\left[\begin{array}{c}
2 \\
-2 \\
-x_{3}-1+x_{2} \\
-3
\end{array}\right], \quad l:=[g, k]=\left[\begin{array}{r}
0 \\
0 \\
-2 \\
0
\end{array}\right] \\
& p:=[f, k]=\left[\begin{array}{c}
4 \\
1 \\
-x_{2}-3 x_{3}+x_{4}+x_{2} x_{3}-\left(1-x_{2}\right)\left(-x_{3}-1+x_{2}\right) \\
9
\end{array}\right] .
\end{aligned}
$$

In $x=0$ we have

$$
\operatorname{rank}[g, k, l, p](0)=4,
$$

and so, the system is accessible.

We will now construct a stable foliation starting from $S_{0}$. Let

$$
S_{s, t}:=l^{s} \circ g^{t}\left(S_{0}\right)
$$


Since $S_{0}=\left\{\left(x_{10}, x_{20}, 0,0\right)^{\mathrm{T}} \mid x_{10} \in \mathbb{R}, x_{20} \in \mathbb{R}\right\}, S_{s . t}$ is given by

$$
S_{s, t}:=\left\{\left(t+x_{10},-t+x_{20}, t-2 s, t\right)^{\top} \mid x_{10} \in \mathbb{R}, x_{20} \in \mathbb{R}, t, s \in \mathbb{R}\right\} .
$$

Obviously, the set $\left\{S_{s, t} \mid s, t \in \mathbb{R}\right\}$ gives a foliation in $\mathbb{R}^{4}$. The distribution $\Delta$ associated with it is $\Delta=\operatorname{sp}\left\{\partial / \partial x_{1}, \partial / \partial x_{2}\right\}$. Unfortunately, this $\Delta$ is not invariant under $f$ as follows from

$$
\left[f, \frac{\partial}{\partial x_{2}}\right]=\left[\begin{array}{c}
0 \\
1 \\
x_{3} \\
0
\end{array}\right] \notin \Delta \quad\left(\text { unless } x_{3}=0\right) \text {. }
$$

This implies that $\Delta_{s}^{*}$ has dimension one at most. Since $\operatorname{sp}\left\{\partial / \partial x_{1}\right\}$ is invariant under $f$ and $g$ and has stable manifold $S_{0}^{1}=\left\{x \mid x_{2}=x_{3}=x_{4}=0\right\}$ through $x=0$ it follows that $\Delta_{\mathrm{s}}^{*}=\operatorname{sp}\left\{\partial / \partial x_{1}\right\}$.

Now consider the dynamics modulo $\Delta_{s}^{*}$. This dynamics is given by $(d \equiv 0)$

$$
\dot{\bar{x}}=\bar{f}(\bar{x})+\bar{g}(\bar{x}) u
$$

where

$$
\bar{f}(\bar{x})=\left(\begin{array}{c}
-x_{2}+x_{4} \\
x_{3}-x_{2} x_{3} \\
3 x_{4}
\end{array}\right), \quad \bar{g}(\bar{x})=\left(\begin{array}{r}
-1 \\
1 \\
1
\end{array}\right) .
$$

It can easily be verified that the pair $((\partial \bar{f} / \partial \bar{x})(0), \bar{g}(0))$ is controllable. This implies that the DDPS for this system is locally solvable if $e \in \Delta_{\mathrm{s}}^{*}=\operatorname{sp}\left\{\partial / \partial x_{1}\right\}$.

\section{Conclusions}

In this section we want to compare our results to those derived by Byrnes \& Isidori [2]. They consider a smooth (i.e. $C^{\infty}$ ) system of the form (2.1). Under certain conditions they solve the Disturbance Decoupling Problem with BIBO-stability.

If we focus our attention to local results for the time being (ignoring the global aspects of the solution in [2]) we see that Byrnes \& Isidori have three important conditions that have to be fulfilled in order to solve this problem, namely they assume that (A1) and (A3) hold and:

(H4) The system (2.1) is exponentially minimum phase.

I.e., $x=0$ is an exponentially stable equilibrium point of the zero dynamics. This is a strong condition, as can easily be seen if the system (2.1), (2.2) is linear. As noted earlier, under the assumption (A3), $\mathscr{R}^{*}=0$ and so for a linear system the zero dynamics is equal to $\left.(A+B F)\right|_{\mathscr{r}^{*}}$ where $F$ is such that $(A+B F) \mathscr{V}^{*} \subset \mathscr{V}^{*}$. The minimum-phase condition implies that $\sigma\left(\left.(A+B F)\right|_{\mathscr{V}^{*}}\right) \subset \mathbb{C}^{-}$, hence $\mathscr{V}_{s}^{*}$ equals $\mathscr{V}^{*}$. (Also in case $\mathscr{R}^{*} \neq 0,(\mathrm{H} 4)$ is equivalent to the equality of $\mathscr{V}_{\mathrm{s}}^{*}$ and $\mathscr{V}^{*}$.) Since we do not require a condition like (H4) to hold in Section 2, in this sense our results are more general.

The paper [2] has an interesting feature that we do not find back in our treatment, namely the existence of a globally defined feedback. Even if we assume that the characteristic numbers $\rho_{i}(x)$ are constant for all $x$, then still our result is local, because the feedback (2.31) can only assure local stability in general. The search for the existence of globally defined stabilizing feedbacks is an interesting problem.

In order to solve the DDPS in practice the problem of finding an algorithm to calculate $\Delta_{\mathrm{s}}^{*}$ explicitly needs attention. Some other problems that are related to the one we treated in Section 2 are e.g. the definition of $\Delta_{\mathrm{s}}^{*}$ in case the largest controllability distribution in the kernel of the output mapping is not the zero distribution and the Noninteracting Control Problem with Stability. 


\section{Appendix}

Consider a smooth vector field $f: \mathbb{R}^{n} \rightarrow \mathbb{R}^{n}$ with $f(0)=0$. If the Jacobian $D f(0)$ has no eigenvalues on the imaginary axis then $f$ is called hyperbolic. For such a vector field $f$ the following theorem holds.

Theorem (Hartman [4]). In a neighborhood of $x=0$ there exist uniquely defined stable and unstable manifolds $S_{\mathrm{s}}$ and $S_{\mathrm{u}}$ that are invariant under $f$ with the same dimensions $n_{\mathrm{s}}$ and $n_{\mathrm{u}}$ as the stable and unstable subspaces $W_{\mathrm{s}}$ and $W_{\mathrm{u}}$ of the system $\dot{z}=D f(0) z$, while in $x=0, S_{\mathrm{s}}$ and $S_{\mathrm{u}}$ are tangent to $W_{\mathrm{s}}$ and $W_{\mathrm{u}}$, respectively. Moreover, if $f$ is $C^{\infty}\left(\mathrm{C}^{\omega}\right)$, then so are $S_{\mathrm{s}}$ and $S_{\mathrm{u}}$.

In Section 2 we sometimes call a manifold $S \subset S_{\mathrm{s}}$ of dimension strictly less than $n_{\mathrm{s}}$ a stable manifold if $S$ is invariant under $f$ and the tangent space of $S$ in $x=0$ is an invariant subspace of the system $\dot{z}=D f(0) z$. It is clear from the context what is meant. A foliation is called stable (unstable) if the leaf through $x=0$ is a stable (unstable) manifold of some known hyperbolic vector field $f$. Furthermore, a vector field $g$ on a manifold $M$ is called transversal to a submanifold $S$ of $M$ if $g(x)$ is not tangent to $S$ at $x$ for all $x \in S$. Finally, the vector fields $\left\{X_{i} \mid i=1, \ldots, p\right\}$ on a manifold $M$ are called independent if $\operatorname{dim} \operatorname{sp}\left\{X_{i}(x) \mid i=1, \ldots, p\right\}=p$ for all $x \in M$.

\section{Acknowledgements}

The authors want to thank Dr. Arjan van der Schaft for many helpful suggestions and for his help during the preparation of this paper.

\section{Literature}

[1] D. Aeyels, Local and global stabilizability for nonlinear systems, in: C.I. Byrnes and A. Lindquist, Eds., Theory and Applications of Nonlinear Control Systems (North-Holland, Amsterdam, 1986) 93-105.

[2] C.I. Byrnes and A. Isidori, Nonlinear disturbance decoupling with stability, in: Proc. of the 26th Conference on Decision and Control (1987) 513-518.

[3] B. Charlet, Robustness and stability for nonlinear systems decoupling and feedback linearization, Systems Control Lett. 8 (1987) 367-374.

[4] P. Hartman, Ordinary Differential Equations (Wiley, New York, 1964).

[5] A. Isidori, Nonlinear Control Systems: An Introduction, Lecture notes in Control and Information Sciences 72 (Springer, Berlin-New York, 1985).

[6] A. Isidori and J.W. Grizzle, Fixed modes and nonlinear noninteracting control with stability, IEEE Trans. Automat. Control 33 (1988) $907-914$.

[7] V. Jurdjevic and J.P. Quinn, Controllability and stability, J. Differential Equations 28 (1978) 381-389.

[8] H. Nijmeijer, Invertibility of affine nonlinear control systems: a geometric approach, Systems Control Lett. 2 (1982) 163-168.

[9] J. Palis and W. de Melo, Geometric Theory of Dynamical Systems (Springer, New York, 1982).

[10] H.J. Sussmann and V. Jurdjevic, Controllability of nonlinear systems, J. Differential Equations 12 (1972) 95-116.

[11] J. Tsinias, Stabilization of non-linear control systems to subspaces, Internat. J. Control 46 (1987) 529-535.

[12] L.L.M. van der Wegen and H. Nijmeijer, A note on disturbance decoupling with stability for nonlinear systems, in: A. Bensoussan and J.L. Lions, Eds., Analysis and Optimization of Systems, Lecture Notes in Control and Information Sciences 111 (Springer, Berlin-New York, 1988) 115-126.

[13] W.M. Wonham, Linear Multivariable Control: A Geometric Approach (Springer, New York, 1979). 